\title{
Periphere Neuropathie
}

\section{Hoch dosiertes Capsaicin-Pflaster schaltet den Schmerz aus}

- Neuropathische Schmerzen sind besonders schwierig zu behandeln. Eine neue Therapieoption für Patienten mit peripheren neuropathischen Schmerzen ist ein Pflaster mit 8\% Capsaicin (Qutenza ${ }^{\mathrm{TM}}$ ), das bei einmaliger Anwendung bis zu zwölf Wochen wirkt. In den klinischen Studien sprachen bis zu 45\% der Behandelten auf die topische Therapie an.

\section{Neuronen reversibel deaktiviert}

Capsaicin stimuliert den Vanilloidrezeptor 1 (TRPV-1), der von afferenten C- und A $\delta$ Nervenfasern exprimiert wird und bei neuropathischen Schmerzen überaktiv ist. Durch Capsaicin 8\% werden die epidermalen Nervenfasern vorübergehend defunktionalisiert, berichtete Priv.-Doz. Dr. Michael Überall aus Nürnberg. Die Folge ist eine deutliche, bis zu zwölf Wochen anhaltende Schmerzlinderung, wie in klinischen Studien bei insgesamt mehr als 2300 Patienten mit Mononeuropathien belegt wurde.
Zugelassen ist das hoch dosierte Capsaicinpflaster zur Behandlung von Erwachsenen mit postherpetischer und HIV-Neuropathie, die nicht an Diabetes erkrankt sind. In einer Zulassungsstudie bei 402 Patienten mit Postzoster-Neuralgie (PZN) nahmen die Schmerzen in der Verumgruppe bei $44 \%$ der Patienten um mindestens $30 \% \mathrm{ab}$; in der aktiven Kontrollgruppe, die einmalig ein Pflaster mit 0,04\% Capsaicin erhielten, sprachen $33 \%$ auf die Therapie an $(p=$ 0,05). Ähnlich wirksam war das Pflaster bei

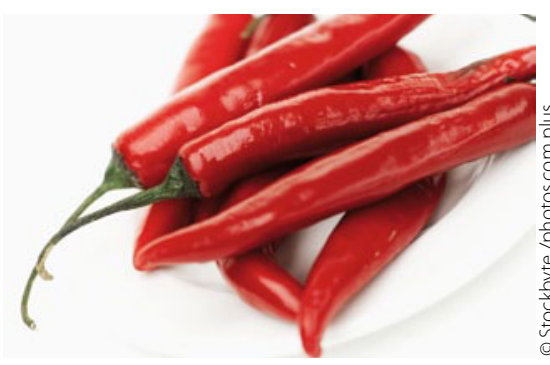

Capsaicin ist ein Inhaltsstoff der Chilischote.
Patienten mit HIV-assoziierter Neuropathie. Die schmerzlindernde Wirkung war bereits in der ersten Woche festzustellen.

Die topische Therapie ist gut mit oralen Medikamenten zu kombinieren und verursacht keine systemischen Nebenwirkungen. Häufigste Nebenwirkungen sind Erytheme und lokale Brennschmerzen bei jeweils etwa $40 \%$ der Patienten.

Nach Vorbehandlung mit 4\% Licocain wird das Pflaster an den Extremitäten für 30 Minuten und am Rumpf für eine Stunde appliziert, berichtete Dr. Thomas Cegla aus Wuppertal. Danach muss mit Kompressen gekühlt werden. Der Anästhesist hat u.a. vier Patienten mit PZN mit dem Pflaster behandelt. Bei dreien waren die Schmerzen nach vier Wochen um mindestens $50 \%$ verringert, ein Patient hatte keine Schmerzen mehr.

- Roland Fath

Quelle: Symposium „Neuropathischer Schmerz - häufig unterschätzt?!", Deutscher Schmerzkongress, Frankfurt (unterstützt von Astellas)

\section{Langzeittherapie der postmenopausalen Osteoporose Wirksame Alternative zu Bisphosphonaten}

— Wichtigstes Behandlungsziel bei Osteoporose ist ein langfristiger Schutz vor Wirbel- und Hüftfrakturen. Eine wirksame und sichere Alternative zur Standardtherapie mit Bisphosphonaten ist der Einsatz von Strontiumranelat (SR).

\section{Störung der Regulationsprozesse}

Die meisten spezifischen Osteoporosemedikamente wirken über eine Verringerung der Knochenresorption, sagte Prof. Franz Jakob, Würzburg. Dieser einseitige Wirkansatz könne auf lange Sicht Probleme mit sich bringen. „Bei der Regulation des Knochenumsatzes hat jede Zelle ihren Platz", erklärte Jakob. So liefern Osteoklasten auch wichtige stimulatorische Impulse für die Knochenneubildung. "Nehmen wir diese Zellen durch eine antiresorptive Therapie aus dem Spiel, stören wir die Signaltransduktion." Dies komme gerade bei alten
Osteoporosepatienten zum Tragen, die in ihrer Regenerationsfähigkeit eingeschränkt seien. Jakob rät deshalb, nach langjähriger antiresorptiver Therapie eine Umstellung auf alternative Wirkstoffe zu erwägen.

Im Gegensatz zu Bisphosphonaten zeigt SR (PROTELOS ${ }^{\circledR}$ ) neben seinem hemmenden Einfluss auf die Knochenresorption in vitro auch eine knochenaufbauende Aktivität. Dies führt zu einer Neueinstellung des Gleichgewichts zugunsten der Knochenbildung.

Nach Auffassung von Prof. Dieter Felsenberg, Berlin, sollte man bei der Wahl des Osteoporosetherapeutikums auch dessen Einfluss auf die Biomechanik des Knochens berücksichtigen. So scheint ein Zuwachs an kortikalem Knochen entscheidend für dessen Biegefestigkeit zu sein. Erste Untersuchungen mit hochauflösender CT hätten gezeigt, dass die Dicke der Kortikalis nach zwölfmonatiger SR-Therapie im Vergleich zu Alendronat deutlich zugenommen hat.

\section{Stabiler Frakturschutz über zehn Jahre}

Dass SR langfristig einen guten und sicheren Frakturschutz bietet, zeigen Erfahrungen über mittlerweile zehn Jahre. Wie Prof. Andreas Kurth, Mainz, berichtete, ist die Inzidenz vertebraler und nicht vertebraler Frakturen in der offenen Verlängerung der Zulassungsstudien gegenüber den ersten Therapiejahren, ungeachtet des erhöhten Altersrisikos, annähernd konstant geblieben. Kurth beurteilte SR in der Langzeit- und Sequenztherapie als gute Alternative zu Bisphosphonaten.

- Dr. med. Martina-Jasmin Utzt Quelle: Satellitensymposium, Osteologiekongress, Fürth (Veranstalter: Servier) 